\title{
Reflexiones sobre el alcance del Plan Distrital de Lectura y Escritura IED Villas del Progreso ${ }^{1}$
}

\section{Reflections on the scope of districts' Reading and Writing Plan, IED Villas del Progreso}

\author{
Reflexões sobre o escopo do Plano de Leitura e Escrita do \\ IED Villas del Progreso
}

\section{Doris Adriana Páez Vigoya²}

Recibido: 9 de junio de 2020 Aprobado: 27 de junio de 2020 Publicado: 3 de agosto de 2020

Cómo citar este artículo:

Páez Vigoya, D. A. Reflexiones sobre el alcance del Plan Distrital de Lectura y Escritura IED

Villas del Progreso. Rastros Rostros, 21(1), 1-18.

doi: https://doi.org/10.16925/2382-4921.2019.01.03

Artículo de investigación. https://doi.org/10.16925/2382-4921.2019.01.03

1 Este artículo de reflexión surge a partir del proceso de intervención pedagógica que se realizó en la IED Villas del Progreso en relación con la Política Pública Distrital "Leer es Volar", que se llevó a cabo entre los años 2017 y2018 en Bogotá.

2 Magíster en Pedagogía de la Lengua Materna. Universidad Distrital Francisco José de Caldas. Docente de Secretaría de Educación del Distrito Capital

Correo electrónico: adrianapaezvigoya@gmail.com

ORCID: https://orcid.org/0000-0002-0340-1796 


\section{Resumen}

Este artículo tiene el propósito de dar a conocer el alcance que tuvo la implementación del Plan Distrital de Lectura y Escritura (PDLE) en Bogotá y específicamente el proceso de intervención pedagógica realizado en la IED Villas del Progreso, ubicada en la localidad de Bosa.

El PDLE surgió en el año 2016, una iniciativa de la Secretaría de Educación del Distrito (SED) como alternativa para mejorar los procesos de lectura y escritura de los niños, y planteó acciones de intervención para los estudiantes de grado tercero en las instituciones con bajo desempeño en las Pruebas Saber de los años 2012, 2014 y 2016. A partir del desarrollo del plan de intervención y acompañamiento pedagógico se propuso llevar a cabo una fase de diagnóstico con los estudiantes para reconocer las dificultades en habilidades comunicativas y, posteriormente, revisar los objetivos propuestos a lo largo de la implementación para conocer el impacto del PDLE in situ. A partir de esta experiencia se reestructuró el plan de estudios de la asignatura de Lenguaje que contribuyó a mejorar los diseños pedagógicos de los docentes y fortaleció la formación profesional atendiendo a las necesidades propias del contexto sociocultural de la institución. En consecuencia, este artículo busca presentar los tipos de participación que se dieron en el marco de la intervención y del proyecto. De igual forma, pretende valorar los alcances tanto de los procesos de formación docente como de las experiencias de aula.

Palabras clave: escritura, lectura, política pública, práctica pedagógica.

\section{Abstract}

The purpose of this article is to publicize the scope of the implementation of Plan Distrital de Lectura y Escritura (PDLE) in Bogotá, specifically the educational intervention process carried out at the Villas del Progreso IED, located in Bosa.

The PDLE emerged in 2016 from the SED as an alternative to improve the reading and writing processes of children, and proposed intervention actions for third grade students in institutions with low performance on the 2012, 2014 and 2016 in Pruebas Saber. From the development of the intervention plan and pedagogical accompaniment, it was proposed to carry out a diagnostic phase with the students to recognize the difficulties in communicative abilities, later, to review the proposed objectives throughout the implementation to know the impact of the PDLE in situ. Based on this experience, the curriculum of the language subject was restructured, which helped to improve the pedagogical designs of teachers and strengthened professional training by addressing the needs of the socio-cultural context of the institution. Consequently, this article seeks to present the types of participation that took place in the framework of the intervention and the project. Similarly, it aims to assess the scope of both teacher training processes and classroom experiences.

Keywords: Writing, reading, political public, pedagogical practice.

\section{Resumo}

O objetivo deste artigo é ao dado publicidade o alcance da implementação de Plan Distrital de Lectura y Escritura (PDLE) em Bogotá, especificamente o processo de intervenção educativo executado no Villas del Progreso IED, localizado em Bosa.

O PDLE emergiu em 2016 do SED como uma alternativa para melhorar a leitura e escritas de crianças e ações de intervenção propostas de estudantes de terceiro ano em instituições com a realização baixa em 2012, 2014 e 2016 no Pruebas Saber. Do desenvolvimento do plano de intervenção e acompanhamento pedagógico, propôs-se executar uma fase diagnóstica com os estudantes para reconhecer as dificuldades em capacidades comunicativas, depois, rever os objetivos propostos durante a implementação para saber o impacto do PDLE in situ. Baseado nesta experiência, o currículo do sujeito de língua reestruturou-se, que ajudou a melhorar os 
desenhos pedagógicos de professores e treinamento profissional fortalecido dirigindo as necessidades do contexto sociocultural da instituição. Consequentemente, este artigo procura apresentar os tipos da participação que se realizou na armação da intervenção e o projeto. Semelhantemente aspira a avaliar o alcance tanto de processos pedagógicos como de experiências de sala de aula.

Palavras-chave: escrita, leitura, prática pública, pedagógica política.

\section{Introducción}

Los procesos de alfabetización han estado presentes desde mucho tiempo atrás, la sociedad en general ha sido responsable de garantizar a los niños y las niñas desde muy temprana edad el aprendizaje de habilidades como la lectura y la escritura. No obstante, en términos de cifras y estadísticas, la responsabilidad recae en las entidades de carácter gubernamental. A nivel latinoamericano, el Centro Regional para el Fomento del Libro en América Latina y el Caribe (CERLALC) es la entidad que se encarga de gestionar planes de alfabetización en países como Colombia, Argentina, Bolivia, Brasil, entre otros, en donde año tras año se realizan pruebas de suficiencia en instituciones educativas y en todos los ciclos de escolaridad. Las pruebas, con niveles de complejidad diferente, buscan hacer medible la capacidad de aprendizaje de los niños y los avances en su proceso escolar; es allí donde surge la pregunta: ¿son los diagnósticos, las pruebas y los estándares una garantía para hacer mejores procesos de enseñanza-aprendizaje en habilidades del lenguaje? Las respuestas al respecto son múltiples y se abordarán en el desarrollo del artículo.

El Plan Distrital de Lectura y Escritura es una estrategia que se articuló con el Plan Nacional de Lectura "Leer es mi cuento" en el año 2016, dando alcance a las metas propuestas por CERLALC y donde dichas orientaciones se dan en relación con la cultura escrita y el acceso a la lectura. El PDLE se planteó un objetivo fundamental, a saber: "promover la lectura y la escritura desde la primera infancia en garantía de los derechos culturales y educativos de los niños y niñas de Bogotá" (2016, p. 5). La estrategia encuentra su campo de acción a partir de tres líneas: la primera consistió en garantizar y estimular las capacidades y el gusto por la lectura y la escritura desde la primera infancia y a lo largo de la vida; la segunda, en fortalecer, modernizar y articular el sistema de bibliotecas públicas escolares y comunitarias y la tercera línea se enfocó en generar conocimiento e intercambio de saberes en torno a prácticas de lectura y escritura. Cada línea planteó acciones que impactaron a la comunidad de la IED Villas del Progreso y posibilitaron diversos niveles de participación en cada grupo de trabajo (docentes, administrativos y estudiantes). 
Así pues, la labor, tanto de intervención como de participación, con la comunidad educativa fue sistematizada pensando en la importancia de recuperar no solamente las propuestas de formación docente, sino de contrastarlas con las concepciones y las voces directas de los participantes. Por lo tanto, este artículo se centra en un proceso de sistematización de prácticas que, según Jara (2006), tiene el propósito de recabar y analizar experiencias entendidas como procesos complejos en donde intervienen una serie de factores objetivos y subjetivos que están en interrelación permanente.

\section{Caracterización del lugar y la población con la que se realizó la intervención}

El proceso de intervención del PDLE en la IED Villas del Progreso, que inició en el año 2017, fue orientado por una asesora pedagógica de la Secretaría de Educación del Distrito. De igual forma, participaron los docentes directores de curso en grado tercero de la jornada de la mañana y de la tarde, de las sedes A y B, cuatro coordinadores y el rector de la institución, cuya participación fue un factor determinante en cada una de las fases de intervención. Los espacios dispuestos para dicha participación estaban distribuidos en dos sedes de la siguiente manera: sede A, ubicada en el barrio Villas del Progreso, donde se trabajó con dos grupos de tercero; mientras la sede B, ubicada en el barrio Bosa Santafé, contaba con cuatro grupos de tercero en la jornada de la mañana y tres grupos en la de la tarde. Los estudiantes de ambas sedes estuvieron acompañados por nueve docentes con las siguientes características de formación académica: dos licenciados en primaria, dos en lengua castellana, dos licenciados en pedagogía infantil y tres en básica primaria con énfasis en matemáticas, lengua castellana e inglés; este grupo de docentes a su vez recibió el respaldo de cuatro coordinadores y el rector de la institución.

En el año 2017 hubo un cese de actividades en el magisterio durante aproximadamente dos meses debido a marchas y protestas en favor de la educación pública. Por lo tanto, la intervención inició de manera tardía en el mes de julio. Inicialmente la asesora del PDLE se entrevistó con el rector para dar a conocer el plan de intervención en la IED y así estableció un cronograma de encuentros y actividades para trabajar con los docentes. En ese sentido, la colaboración de los coordinadores fue decisiva pues era necesario que los docentes de grado tercero contaran con tiempo y espacios para dialogar sobre el plan de intervención y pudieran asistir a las capacitaciones organizadas por la Secretaría de Educación del Distrito. 


\section{Línea 1 del proyecto PNLE: garantizar y estimular las capacidades y el gusto por la lectura y la escritura}

La primera línea de trabajo propuesta por el PDLE consistía en garantizar y estimular las capacidades y el gusto por la lectura y la escritura, pues el objetivo era lograr que todos los niños y las niñas de grado tercero de la IED estuvieran en capacidad de leer y escribir a los 8 años y así garantizarles una trayectoria escolar exitosa. El reto consistía en disminuir de $45 \%$ a $35 \%$ el porcentaje de estudiantes con niveles de desempeño insuficientes o mínimos en la Prueba Saber $3^{\circ}$.

En consecuencia, para cumplir con la primera línea de trabajo, se acordó un encuentro institucional para revisar los resultados de la Prueba Saber de los años 2012 a 2016 con el objetivo de identificar el nivel de las competencias que evalúa el ICFES en el área lenguaje para grado tercero.

\section{El diagnóstico}

Así las cosas, se analizaron los resultados de 362 estudiantes que presentaron la prueba en 2016 y se evidenciaron los siguientes aspectos a propósito de las competencias evaluadas en la prueba. En la competencia escritora se constata que del $100 \%$ de los estudiantes, el $49 \%$ no contestó correctamente; también fue posible identificar que el $80 \%$ de los estudiantes se clasificó en el nivel mínimo y el $20 \%$ en el nivel satisfactorio. Ahora bien, en cuanto a la competencia lectora, el $45 \%$ de los estudiantes no contestó correctamente las preguntas; el $13 \%$ de las respuestas se clasificó en el nivel insuficiente, mientras que el $63 \%$ en mínimo, el $25 \%$ en satisfactorio y el $0 \%$ en avanzado. En el año 2015 el $46 \%$ de los estudiantes fue clasificado en un nivel de desempeño mínimo e insuficiente, mientras que en el año 2016 hubo un incremento desfavorable del 51 \% de estudiantes en nivel mínimo e insuficiente.

A partir de los datos anteriormente relacionados se evidencia la problemática en habilidades básicas de lectura y escritura de la población estudiantil en Pruebas Saber que evalúan de forma estandarizada. Luego de revisar los resultados de la evaluación externa Prueba Saber $3^{\circ}$ se dio a conocer una prueba diseñada por la SED para aplicar en los colegios focalizados que evaluaba habilidades en lectura y escritura; además, era aplicada exclusivamente por la asesora pedagógica priorizando procesos básicos de identificación de micro y macroestructuras de textos escritos, lectura en voz alta y comprensión de tipo literal. Al tratarse de una prueba con carácter diagnóstico se realizó con 374 estudiantes en el mes de julio de 2017. Cabe aclarar 
que la prueba evaluaba 19 aspectos relacionados con habilidades comunicativas, entre ellos el reconocimiento de palabras como unidad gráfica, la identificación de sílabas en palabras, la identificación del asunto principal del texto, la toma de dictado sin errores ortográficos, la formulación de hipótesis de lectura y escritura de un texto corto descriptivo, entre otras. Los niveles de desempeño para la prueba estaban clasificados en avanzado, satisfactorio, mínimo e insuficiente. Entre los hallazgos se encontró que la mayoría de los estudiantes que presentaron la prueba se ubicaron en los desempeños mínimo e insuficiente; del total de los niños que presentaron la prueba interna solamente 17 se ubicaron en el nivel satisfactorio y un estudiante en el nivel avanzado.

Predomina, por lo tanto, el desempeño bajo e insuficiente en la mayoría de las habilidades evaluadas. Se identificó que más de 200 estudiantes tenían dificultad en diferenciar números de letras, reconocer la palabra como unidad gráfica, identificar espacios entre palabras y puntuación, identificar el sonido de la sílaba al final de la palabra y la cantidad de sílabas en una palabra; así como el número de palabras en una frase, la tipología textual y la formulación de hipótesis sobre el texto.

Entre tanto, las habilidades en las que los estudiantes tuvieron desempeño satisfactorio o avanzado fueron leer en voz alta y con fluidez palabras, frases o textos; identificar la idea principal en el texto; localizar información explícita; reconocer la intención comunicativa; establecer inferencias; escribir sin errores ortográficos y escribir un texto corto descriptivo.

Ahora bien, otros aspectos que se tuvieron en cuenta en la prueba de diagnóstico se sitúan en la identificación de los estudiantes con capacidades diversas que no leen ni escriben. Así como los casos de bajo rendimiento que, aunque no estén diagnosticados como población de inclusión, manifiestan dificultades cercanas a este grupo poblacional pues en estos estudiantes se evidencia la falta de autonomía para desarrollar actividades debido a la dificultad para comprender instrucciones escritas y orales; mientras otros estudiantes reportaban inasistencias frecuentes. Por último, se evidenció poca disposición de algunos docentes para acompañar la aplicación del diagnóstico.

Bajo ese panorama, las metas institucionales que se planearon con base en los resultados arrojados en la prueba diagnóstica para los años 2017 y 2018 estuvieron encaminadas a posibilitar que los estudiantes, a través de metodologías y estrategias, consolidaran en un $100 \%$ las habilidades en las que presentaban dificultades respecto a la apropiación de escritura, lectura y producción de textos. A su vez, se propuso plantear una estrategia conjunta que pudiera atender a los estudiantes que presentaban mayor dificultad en cada uno de los grupos. De igual forma, se planteó 
ayudar a reducir los desempeños insuficiente y mínimo de un $60 \%$ a un $55 \%$ en las pruebas Saber $3^{\circ}$ para el año 2018. En consecuencia, requeriría de un monitoreo constante sobre el avance de los estudiantes con respecto a la comprensión de lectura y producción de textos; esto con el fin de identificar las habilidades específicas a ser trabajadas y el nivel de desempeño alcanzado por cada uno de ellos, considerando sus características y las acciones de intervención pedagógica necesarias para atenderlos de mejor manera. Entre otras cosas fue necesario facilitar espacios de trabajo pedagógico para potenciar el nivel de actualización disciplinar de los docentes y dar respuesta a las dificultades de aprendizaje.

En cada uno de los nueve grupos en los que se hizo el diagnóstico fue posible caracterizar aquellos estudiantes que presentaban mayor dificultad. Así se planteó una ruta de trabajo en la que se describían las habilidades de mayor atención para que los docentes trabajaran en ello, también incluyó el nombre de los niños, las estrategias que se iban a utilizar, las metas propuestas, el tiempo para ejecutar las actividades y los responsables de lograrlo.

\begin{tabular}{|c|c|c|c|c|c|}
\hline $\begin{array}{c}\text { Ouob } \\
\text { (Capacidados) }\end{array}$ & $\begin{array}{c}\text { Quibn } \\
\text { (Estudiantos) }\end{array}$ & $\begin{array}{c}\text { Cómo } \\
\text { (Estratogia) }\end{array}$ & $\begin{array}{l}\text { ¿Para quô? } \\
\text { (Mota) }\end{array}$ & $\begin{array}{c}\text { Cuándo } \\
\text { (Tiompo/periodo) }\end{array}$ & Rosponsablo \\
\hline $\begin{array}{l}\text {-Comprender } \\
\text { diferencias entre la } \\
\text { escritura alfabetica } \\
\text { y otras formas } \\
\text { graficas. (APRO- } \\
\text { SIST-ESCRIT) } \\
\text { - Comprender la } \\
\text { función de } \\
\text { segmentacion de } \\
\text { los espacios en } \\
\text { blanco y de la } \\
\text { puntuacion final de } \\
\text { la frase. (APRO- } \\
\text { SIST-ESCRIT) } \\
\text { - Reconocer } \\
\text { unidades } \\
\text { fonologicas como } \\
\text { sinabas, rimas. } \\
\text { terminaciones de } \\
\text { palabras, etc. } \\
\text { (APRO.SIST- } \\
\text { ESCRIT) } \\
\text { - Comprender la } \\
\text { naturaleza } \\
\text { altabetica del } \\
\text { sistema de } \\
\text { escritura. (APRO. } \\
\text { SIST-ESCRIT) }\end{array}$ & $\begin{array}{l}\text { 303-MANANA } \\
11 \text { estudiantes } \\
\text { 304-MANANA } \\
\text { 16 estudiantes }\end{array}$ & 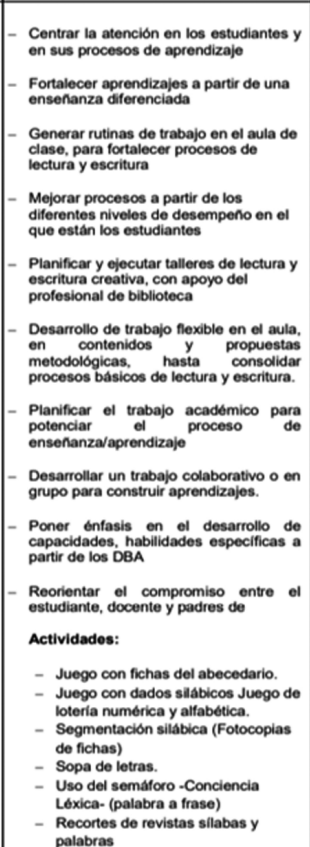 & $\begin{array}{l}\text { - Monitorear constantemente ef } \\
\text { avance de los estudiantes con } \\
\text { respecto a la comprension de } \\
\text { lectura y produccion de textos. } \\
\text { - Hacer seguimiento semanal del } \\
\text { proceso de avance de cada nino. } \\
\text { - Fomentar la rigurosidad } \\
\text { académica en el aula de clase. } \\
\text { - Fortalecer } \\
\text { desempenos/capacidades } \\
\text { particulares en los estudiantes } \\
\text { - Fomentar hábitos lectores y } \\
\text { escritores en espacios diferentes } \\
\text { al aula de clase y con difterentes } \\
\text { Ilderes. } \\
\text { - Priorizar la consolidacion de } \\
\text { aprendizajos no consolidados. } \\
\text { - Posicionar a los estudiantes como } \\
\text { eje principal de su aprendizaje. } \\
\text { - Incentivar el trabajo con el par } \\
\text { académico, ser propositivo, y } \\
\text { atento a las sugerencias y } \\
\text { acciones de cambio. } \\
\text { - Tener clara las directrices o } \\
\text { elementos generales para la } \\
\text { apropiacion de capacidades } \\
\text { desde lineamientos educativos } \\
\text { distritales y nacionales }\end{array}$ & 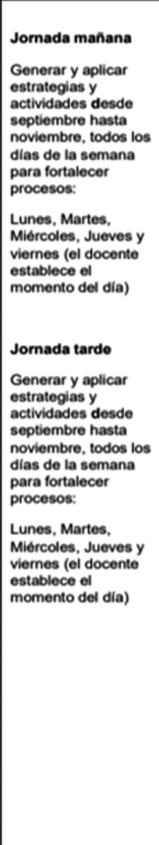 & 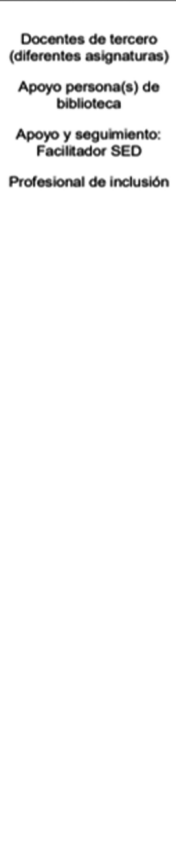 \\
\hline
\end{tabular}

Figura 1. Cuadro de caracterización de los grupos de grado tercero Fuente: Secretaría de Educación del Distrito (SED), 2017. 
Frente al diseño de este plan de intervención institucional se evidenció que había toda una propuesta de trabajo por parte de la SED en la que no se quería dejar ninguna situación al azar. Este plan fue presentado a los docentes, coordinadores y rector de la institución pero se identificaba una limitación relacionada con el tiempo que se tenía para trabajar, esto por dos razones: la primera asociada con la cantidad de actividades y proyectos en los que el colegio ya venía trabajando, a saber, proyectos de ciudadanía y convivencia, educación sexual, jornada extendida, ajedrez en el aula y la segunda estaba relacionada con el momento del año en que la información fue presentada (agosto de 2017), pues se estaba a dos meses de culminar el año escolar. A pesar de todo, con los avances a la fecha y con ciertas reticencias por parte de algunos docentes se puso en marcha la propuesta. Se trataba de una intervención que venía muy bien planeada y aunque algunos docentes lo veían como una imposición, para el rector y los coordinadores era una oportunidad de sacar el mejor provecho tanto del material y el tiempo como de las herramientas que estaba brindando la SED. La expectativa era muy alta, pero al mismo tiempo implicaba un compromiso total por parte de los docentes, finalmente eran ellos quienes asumían la ejecución y distribuían el tiempo de la jornada para trabajar en las actividades previamente diseñadas.

\section{Percepción de docentes}

Frente a esta perspectiva de trabajo, los nueve docentes que participaron en la intervención del PDLE se mostraron interesados por mejorar los resultados de la Prueba Saber $3^{\circ}$ y a su vez por los resultados de las pruebas de diagnóstico que se hicieron en la institución. Sin embargo, tres de ellos manifestaron que la propuesta estaba enfocada en dar cifras y resultados a corto plazo de procesos bastante complejos como la lectura y la escritura. Las opiniones entre los docentes estuvieron divididas, mientras que unos aseguraban su pertinencia otros opinaban lo contrario; pese a las diferencias entre maestros es importante reconocer que la propuesta tenía un valor en sí misma y la labor de la institución y de los profesores consistía en sacar el mejor provecho de esta elección.

Algunos docentes insistían en que los planes de intervención buscaban dar resultados a corto plazo sin tener en cuenta las condiciones sociales, culturales y académicas de los niños. Si bien es cierto que Yepes (2013) define la lectura como un proceso que sobrepasa la decodificación así el individuo puede hacer con la lectura elaboraciones conceptuales, argumentar ideas, comprender y comunicar, también requiere de otras lecturas distintas de la palabra escrita; de modo que algunos docentes apelaban a esta mirada, por lo tanto, tomaron distancia de la propuesta y 
decidieron continuar con sus clases habituales, considerando que la intervención no sería suficiente.

Otro grupo de maestros aseguraba no estar de acuerdo con la medición estandarizada, es decir, desde su perspectiva se consideraba que la intervención solamente tendría lugar para obtener mejores resultados en la Prueba Saber; en este sentido, lo asumieron como un mecanismo de presión para obtener resultados a corto plazo, argumentando que la necesidad de mostrar evidencias era la única preocupación, además veían que no había relación entre el documento de lineamientos y DBA (derechos básicos de aprendizaje) propuestos para el grado tercero. Se hace necesario aclarar que este material es diseñado por el Ministerio de Educación y muestra un perfil de estudiante en el que no se tienen en cuenta las diferencias entre la población escolar y mucho menos los procesos de aprendizaje previos, edades y contextos familiares.

El grupo de maestros restantes - aproximadamente seis- consideraban que un plan de intervención era la oportunidad para mejorar el desempeño en lectura y escritura y de paso reconocer estrategias, metodologías y actividades que fortalecieran las habilidades comunicativas de los estudiantes, siempre que los procesos de formación contribuyeran a replantear el ejercicio docente. Hubo docentes que asumieron posturas favorables puesto que no habían vivido experiencias anteriores con la Secretaría de Educación en el marco de la política pública y para ellos era novedosa la propuesta porque implicaba tres fases, una de diagnóstico, otra de intervención y una de análisis. En este ejercicio de reflexión se evidenció que la comunidad docente participó de diversas maneras incluso con posturas divididas entre ellos.

En la sede A de la institución las docentes aseguraban que "si ya nos dijeron qué dificultades tienen los niños, ya los tenemos focalizados y además nos van a dar material para trabajar, tenemos que trabajar en equipo, ojalá antes de terminar el año escolar" (C. Rodríguez, comunicación personal, 12 de mayo de 2020). Por su parte, las docentes de la sede $B$ de la jornada de la mañana manifestaron que las temáticas trabajadas en los procesos de capacitación eran pertinentes y de gran relevancia; sin embargo, estos espacios de formación no debían ser exclusivos para docentes de tercer grado, era importante vincular a las docentes de primero y segundo quienes anteceden la iniciación a los procesos de lectura y escritura. ${ }^{7}$

A pesar de las diferencias entre docentes, la asesora pedagógica que acompañó la intervención empezó a visitar las aulas. En algunos momentos los docentes acompañaban el proceso en el aula. En otras ocasiones, los docentes salían del aula

1 Esta opinión hace parte de la encuesta realizada a los docentes de la IED Villas del Progreso que participaron de la intervención. La información se obtiene a través de un formulario de Google 2020 que recopila sus opiniones en relación con los procesos de intervención del PDLE. 
y la asesora asumía el grupo, las actividades que ella planeaba estaban contempladas para desarrollarse en treinta minutos o máximo una hora.

\section{Intervención}

La asesora pedagógica de la SED, luego de hacer el diagnóstico con cada grupo, empezó a realizar el trabajo de intervención; ella llevaba material para trabajar las habilidades comunicativas con los estudiantes de acuerdo con el nivel que habían alcanzado en las pruebas, es decir, guías para los niveles básico, medio, alto. Las actividades contenían ejercicios para fortalecer conciencia fonética y fonológica en los estudiantes. Incluía, además, ejercicios de comprensión de tipo literal e inferencial y actividades para reconocer los personajes en una historia, ideas principales y secundarias de un texto.

Durante el desarrollo de las acciones pedagógicas, hubo un ejercicio que llamó la atención de los niños porque durante una sesión la asesora leyó el libro Donde viven los monstruos de Maurice Sendak (1963) permitiendo que un estudiante representara la actuación del protagonista. Con este ejercicio los niños se mostraron motivados y solicitaron a los docentes realizar ejercicios similares. La docente facilitadora de la SED propició el trabajo en el aula, de tipo práctico y experiencial, a partir del cual el docente se vio en la tarea de revisar nuevas metodologías según las necesidades evidenciadas en el diagnóstico, y la mayoría se animaron a diseñar su propio material. En ocasiones los talleres que diseñaban los maestros se enviaban a la docente facilitadora quien hacía algunas observaciones y comentarios muy asertivos, lo cual contribuyó en gran medida a que los maestros se apropiaran de la intervención.

A pesar de que los profesores diseñaron material, no tuvieron la oportunidad de establecer sesiones de encuentro con sus pares para dialogar sobre lo realizado. Por lo tanto, su labor se presentó como resultado de un trabajo individual. El mayor logro, en este sentido, fue trabajar por sedes y jornadas, pues el trabajo institucional siempre estuvo fragmentado por la dificultad que había para establecer espacios de encuentro entre docentes.

En relación con el tiempo de ejecución del proyecto, cada ocho o quince días la asesora visitaba las diferentes sedes y enviaba material por correo electrónico a los docentes para que diseñaran actividades y todas fueran consecuentes con los propósitos que se habían trazado para cada uno de los grupos. En varias oportunidades la asesora ingresó a los salones de clase para orientar ejercicios de lectura en voz alta, lectura compartida y lectura grupal con los estudiantes. Para la mayoría de los docentes este ejercicio fue dialógico porque ayudó a nutrir las prácticas pedagógicas 
que ya venían realizando, permitió compartir conocimiento práctico sin la pretensión de ostentar manejo de la teoría pedagógica y metodológica.

Por ejemplo, en uno de los grupos la docente titular tomó la decisión de trabajar con un grupo focal de quince estudiantes que aún no desarrollaban procesos básicos de lectura, como silabeo, segmentación de palabras, comprensión literal y lectura en voz alta. El tiempo de la jornada no era suficiente, entonces a través del programa Todos a aprender ${ }^{2}$ tuvo la oportunidad de presentar una propuesta de refuerzo escolar los sábados, distribuido en 10 sesiones con una intensidad de dos horas y eso permitió un trabajo más personalizado con los niños que aún no habían consolidado ciertos procesos tanto en lectura como escritura. Algunos estudiantes además tenían extra-edad, es decir, cursaban tercero y tenían entre 10 y 12 años o habían reiniciado año escolar, su avance fue significativo lo que ayudó a ganar confianza tanto en los estudiantes como en los padres para continuar el proceso.

Aunque de los nueve docentes participantes solamente seis se involucraron de forma activa, se empezaron a observar cambios significativos en los grupos de niños donde habían dedicado tiempo extra para acompañar el proceso. Por ejemplo, algunos solicitaron el acompañamiento de los padres de familia, este aspecto fue decisivo para empezar a notar mejoría en el desempeño de los estudiantes; aunque no todos los padres colaboraron, un buen número de ellos se sintió comprometido con la tarea de reforzar en casa las prácticas de lectura. En consecuencia, la lectura dejó de ser para muchos niños una limitación y se convirtió en la oportunidad para mejorar su desempeño a todo nivel.

Adicionalmente, se asignaban tareas o actividades para hacer refuerzo en casa y con las familias. Si bien con los niños que no recibían acompañamiento en casa el proceso fue más lento, también se lograron avances. Lo anterior permitió establecer espacios de lectura en el colegio en los que participaban padres de familia, algunos niños leían en voz alta y otros hacían preguntas o respondían interrogantes. Al principio los niños no querían leer porque se sentían tímidos e inseguros, pero poco a poco el miedo desapareció y cada uno quería hacer la lectura a su ritmo y había disminuido la tensión por las críticas. Evidentemente, cada niño estaba alcanzado su propia meta de lectura.

2 El programa Todos a aprender tiene entre sus objetivos fomentar espacios de acompañamiento a la población estudiantil de acuerdo con un cronograma y a una agenda con temáticas y objetivos definidos por la SED. 


\section{Línea 2 del proyecto PDLE: fortalecer, modernizar y articular el sistema de bibliotecas públicas escolares y comunitarias}

La segunda línea del PDLE hace referencia al fortalecimiento, modernización y articulación del sistema de bibliotecas escolares. En ese sentido, la IED Villas del Progreso recibió 388 libros de texto en las áreas de matemáticas y lenguaje, para ser distribuidos en los 9 grupos de estudiantes y 11 textos de guía para los docentes con el fin de apoyar el trabajo de intervención que se venía realizando en las aulas. Aquí es importante aclarar que la institución ya contaba con material de lectura adquirido en años anteriores para el proyecto de lectura institucional "10 libros en un año", este material fue bien recibido por los docentes porque incluía guías de trabajo y actividades sobre lectura, escritura y razonamiento para trabajar en clase con los niños y también traía una cartilla de trabajo para que estos llevaran a casa.

Algunos docentes aseguraban que el material de apoyo contribuía al proceso de muchos niños y niñas que no tenían acceso a estos recursos, ya que algunos padres no adquirían los libros propuestos por la institución porque no contaban con el presupuesto, así que tener material impreso y colorido motivaba a los niños a leer. Aunque no se disponía de mucho espacio para guardar el material, cada docente asumió el cuidado de los libros junto con sus estudiantes. Entonces, había días de la semana en los que se trabajaba en el libro, cada niño tenía uno, a veces lo llevaban para la casa y esto era asumido como incentivo, los niños disfrutaban observando las ilustraciones que traían los libros de texto y las actividades que podían desarrollar. La tenencia del libro les generaba sentido de pertenencia y compromiso.

No obstante, la tenencia de este material no constituía una garantía de los aprendizajes. En este sentido, Colomer (2002) afirma que es necesario establecer un puente de comunicación entre la recepción individual hasta la recepción de una comunidad cultural que interprete y dé valor a todo lo que ocurre al interior de la escuela. De modo que el rol del docente mediador y planeador constituía la base de los aprendizajes para los niños. Todo ese material necesitaba ser analizado por los docentes para luego seleccionar las actividades que mejor se ajustaran a las necesidades de los estudiantes. Sin lugar a duda, fue un trabajo arduo y permanente que exigía compromiso total por parte de los maestros.

Los avances que se produjeron sobre la posibilidad de leer en el aula trajeron consigo ambientes más propicios para la lectura ocasionados por los mismos 
estudiantes, una vez el docente iniciaba una lectura había un ambiente de silencio absoluto, los niños tenían el texto y todos podían seguir la lectura, de esta manera, el docente delegaba la lectura en voz alta y algunos estudiantes se motivaban a participar, situación que antes no era frecuente.

\section{Línea 3 del proyecto PNLE: generar conocimiento e intercambio de saberes, en torno a prácticas de lectura y escritura}

\section{Formación docente}

Los procesos de formación para los docentes estuvieron enfocados en tres aspectos: el primero consistía en ofrecer un espacio de sensibilización donde se reconociera la importancia de la labor docente en los procesos de aprendizaje de los estudiantes, un docente que se reconoce como agente transformador de la sociedad va a buscar mecanismos de trabajo que contribuyan al fortalecimiento de las capacidades de sus estudiantes. Así que los encuentros realizados fuera de la institución tenían dos objetivos: por un lado, estaba el grupo de docentes que nunca había participado en procesos de formación de la SED y lo consideraban como un ejercicio liberador, para ellos era reconocer nuevas formas de trabajo en aula, compartir experiencias con maestros de otras instituciones y renovar sus prácticas; por otro lado, para los que habían participado en procesos de formación anteriores se trataba de una intervención que no resultaba innovadora. Algunos explicaban que los procesos de intervención no llegaban a las instituciones para quedarse, al principio se mostraban muy interesantes, pero una vez se entregaban las evidencias del trabajo realizado, el plan de intervención terminaba y las prácticas innovadoras quedaban en el olvido, incluso si no eran sistematizadas no había garantía de sostenibilidad.

El segundo aspecto interesante e influyente en la formación docente consistió en compartir y reflexionar en torno a experiencias exitosas sobre lectura y escritura en países de América Latina como Brasil, Costa Rica, El Salvador, Argentina, esto a través de invitadas expertas, asesoras educativas provenientes de Minas Gerais y el Ministerio de Educación de Brasil -con trayectoria y reconocimiento por más de treinta años- que buscaban establecer diálogo entre colegas para identificar rutas de trabajo, ejes de formación y metodologías que fueran aplicables en los contextos de la ciudad. 
Parecía que las experiencias de las asesoras educativas invitadas eran tan eficaces que incluso en aulas de Colombia, específicamente bogotanas, tendrían lugar; sin embargo, los docentes conocedores de propuestas nacionales, locales e institucionales una y otra vez replicaban en las jornadas de formación que aprender a leer y a escribir no se trataba de una fórmula mágica infalible, más bien de un aprendizaje que se construía a partir de las experiencias propias de cada institución y requería de varios agentes transformadores como la familia, el entorno social, los grupos de compañeros, los contextos sociales, culturales y económicos como espacios de formación. A pesar de lo interesante de los espacios de diálogo entre países, este ejercicio privilegiaba modelos extranjeros que al parecer habían sido diseñados a prueba de todo y no se tenían en cuenta las propuestas a nivel nacional.

El tercer aspecto de formación docente estaba relacionado con actividades específicas de trabajo en aula como conciencia fonética, fonológica, lectura en voz alta, comprensión de tipo literal e inferencial, dirigidas a contrarrestar la problemática sobre las dificultades que mostraban los niños en lectura, escritura e incluso oralidad. Para propiciar los procesos de actualización se entregaron guías de trabajo y talleres, actividades que iban a facilitar al docente establecer el punto de partida para iniciar su propia ruta de trabajo. Aunque no había un formato para diseñar los talleres, algunos docentes habían decidido tomar como ejemplo las actividades que la asesora pedagógica llevaba y hacían sus propias adaptaciones. Eran conscientes de propiciar espacios y planear actividades diferentes que fortalecieran las habilidades básicas y necesarias para lograr mejores procesos en lectura y escritura, lo suficientemente sólidos para garantizar procesos de aprendizaje en los niños.

A pesar de lo interesante que resultó ser la estrategia, el hecho de no tener indicaciones metodológicas y de diseño, de manera explícita, seguía dejando relegado el saber pedagógico a un segundo plano, como si bastara con dar ejemplos para formar a los docentes en diseño curricular; situación que una vez más dejó al descubierto la restringida planeación de la SED en términos de formación pedagógica. Los objetivos de actualización continúan siendo los conceptos de la disciplina, pero no las metodologías específicas.

En la figura 2 se observa la propuesta que planteó la SED en los talleres de formación para docentes. 


\section{Componentes de la enseñanza de la lectura y la escritura en la etapa inicial}

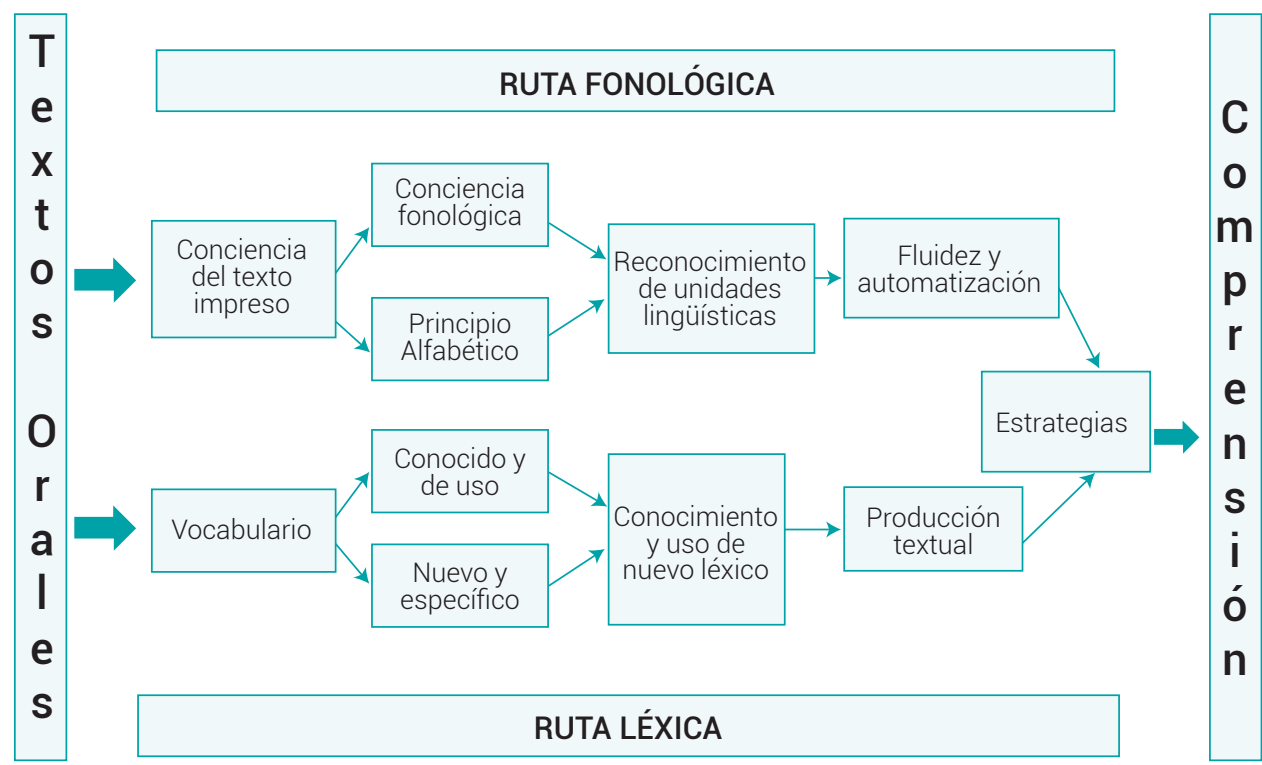

Figura 2. Ruta de enseñanza de la lectura y la escritura en la etapa inicial, presentada por la SED

Fuente: Secretaría de Educación del Distrito (SED), 2017 a.

A través de esta ruta se buscaba orientar el aprendizaje de los niños y las niñas de grado tercero en todas las instituciones de Bogotá, con un diseño metodológico que privilegiaba el desarrollo cognitivo y neuronal dejando de lado las prácticas socioculturales en las que evidentemente los niños también aprenden, incluso en los contextos de mayor vulnerabilidad ellos asimilan más por medio de la imitación de prácticas sociales y culturales que de las prácticas puramente cognitivas. Cassany (2006) asegura que el aprendizaje de las habilidades del lenguaje, incluyendo el discurso, no surgen de la nada, este refleja puntos de vista, visiones de mundo. Es así como cada comunidad se expresa según su identidad y su historia.

De lo anterior se derivan las implicaciones que puede ocasionar el trabajo en el aula basado en propuestas prediseñadas y generalizadas, que no dan paso a una reflexión en la que se sitúen procesos que sean dinámicos y den cuenta de las necesidades en contextos reales en donde se dan esas interacciones. Los procesos de formación anteriormente descritos se realizaban en jornadas de 8 horas diarias durante dos días. A lo largo de la implementación se realizaron tres encuentros muy similares: Ios escenarios de participación colectiva eran la Biblioteca Pública Gabriel García Márquez y un reconocido hotel en Bogotá. Asistían entre 200 y 300 maestros de diferentes instituciones públicas. Allí los docentes recibían atención especial, material 
de trabajo para cada sesión y memorias del evento para que luego fueran socializadas en las instituciones durante las reuniones de ciclo y de área.

Sin lugar a duda, es interesante ver que hubo un esfuerzo por proveer de materiales a los docentes y generar un entorno propicio para el aprendizaje y el trabajo colectivo. Sin embargo, el hecho de trabajar en distintas sedes sin tener la posibilidad de acordar encuentros posteriores debilita las iniciativas y no permite dar continuidad para hacer seguimiento a los procesos ni formas de asesoría que brinden soporte permanente a la labor docente. En consecuencia, existe una necesidad de ocasionar espacios permanentes y agradables dentro y fuera de la institución para que los docentes no solo tengan acceso a capacitación, sino a seguimiento y acompañamiento para no olvidar los procesos que inician bien.

\section{Reflexiones finales}

El proceso de intervención permitió contemplar la posibilidad de hacer ajustes en el plan de estudios de la asignatura Lengua Castellana para el año 2018, pues el trabajo que había realizado cada docente apuntaba a diseñar secuencias didácticas que favorecían el uso de las habilidades lingüísticas; no obstante, era preciso abrir el debate con todos los docentes de lenguaje de la institución y, para ello, resultaba complejo establecer horarios de encuentro. Además, siempre había posiciones divididas frente a propuestas innovadoras, esto se reflejaba en que, si algún docente proponía algo, él o ella debía trabajar en su ejecución pues el trabajo en equipo no había sido fácil. Esta discusión se dio en enero de 2018, luego de ver los resultados de la primera intervención.

Ciertamente, a partir de los resultados obtenidos de las pruebas finales hechas a los estudiantes se observó el avance de la mayoría de los niños en habilidades de lectura y escritura, especialmente en los grupos donde los docentes habían acompañado el proceso de intervención de forma constante. En cambio, en los grupos donde los maestros habían continuado con prácticas habituales no se notaban diferencias. Con ayuda de esta información el rector tomó decisiones sobre la asignación de cursos para el año siguiente. Lo que pretendía el directivo era que los docentes quienes habían llevado a los niños a mejorar su desempeño orientaran clase en grado segundo y, de ese modo, replicaran los diseños pedagógicos y las metodologías que habían sido exitosas en otros cursos.

Ahora bien, la potencialidad de sistematizar este tipo de experiencias dio como resultado la construcción de una propuesta escrita a partir de la vivencia de los docentes, en la que se describía la ruta de trabajo orientadora y que en momentos 
posteriores podía ser utilizada como material de consulta para tomar decisiones, hacer ajustes o retomar posturas que favorecieran el trabajo no solo de la asignatura Lenguaje, sino del trabajo transversal en las demás materias.

A través de esta experiencia de intervención se evidenció que las pruebas, los resultados y las cifras no son suficientes para mejorar procesos en habilidades comunicativas, más bien se consideran un referente para tomar decisiones, son indicadores importantes que permiten reconocer necesidades de capacitación, procesos de cambio y planeación pedagógica que pueden transformar la realidad del aula. Pero existen otros factores externos que no dependen de la planeación escolar, recordemos que la lectura y la escritura son habilidades que transforman y ayudan a generar condiciones para la participación en la vida ciudadana, por ende, se aprenden en todos los escenarios.

Por último, se hizo evidente la necesidad de proponer acciones de mejora con relación a los procesos de intervención que propone la SED para llevar a cabo en las instituciones, con el fin de establecer acuerdos entre lo que se plantea y lo que se ejecuta, el acompañamiento y la puesta en marcha de las propuestas de política pública les ofrece a las instituciones educativas respaldo y credibilidad frente al logro de los objetivos propuestos.

\section{Referencias}

Cassany, D. (2006). Tras las líneas sobre la lectura contemporánea. Editorial Anagrama.

Colomer, T. (2002). La lectura infantil y juvenil En J.A. Milán, La lectura en España. Informe 2002, (pp. 263-285). Federación de Gremios de Editores de España. https://www.academia. edu/38064834/La_lectura_infantil_y_juvenil

Jara, O. (2006). Orientaciones teórico-prácticas para la sistematización de experiencias. Alforja. http://centroderecursos.alboan.org/ebooks/0000/0788/6_JAR_ORI.pdf

Secretaría de Educación del Distrito. (2016). Plan Distrital de Lectura y Escritura (PDLE). https:// www.culturarecreacionydeporte.gov.co/biblioteca/descargables/Plan\%20de\%20lectura_100217.pdf

Secretaría de Educación del Distrito. (2017). Informe de intervención propuesto por la SED Bogotá en la IED Villas del Progreso. 
18 Reflexiones sobre el alcance del Plan Distrital de Lectura y Escritura IED Villas del Progreso

Secretaría de Educación del Distrito (SED). (2017a). Ruta de enseñanza de la lectura y la escritura en la etapa inicial, presentada por la SED. Sesiones de formación para docentes en el marco del Plan Distrital de Lectura y Escritura.

Sendak, M. (1963). Donde viven los monstruos. Editorial Harper Collins.

Yepes, L. (2013). Jóvenes lectores. Caminos de formación. Ministerio de Educación y Cultura del Uruguay. https://cerlalc.org/publicaciones/jovenes-lectores-caminos-de-formacion/ 